\title{
The evolution of the mass-metallicity relations from the VANDELS survey and the GAEA Semi-Analytic model.
}

Fabio Fontanot ${ }^{1,2 \star}$, Antonello Calabrò ${ }^{3}$, Margherita Talia ${ }^{4,5}$, Filippo Mannucci ${ }^{6}$, Marco Castellano ${ }^{3}$, Giovanni Cresci ${ }^{6}$, Gabriella De Lucia ${ }^{1}$, Anna Gallazzi ${ }^{6}$, Michaela Hirschmann $^{7}$, Laura Pentericci ${ }^{3}$, Lizhi Xie ${ }^{8}$, Ricardo Amorin ${ }^{10,11}$, Micol Bolzonella ${ }^{5}$, Angela Bongiorno ${ }^{3}$, Olga Cucciati ${ }^{5}$, Fergus Cullen ${ }^{12}$, Johan P. U. Fynbo ${ }^{13}$, Nimish Hathi $^{14}$, Pascale Hibon ${ }^{15}$, Ross J. McLure ${ }^{12}$, Lucia Pozzetti ${ }^{5}$

\author{
1 INAF - Astronomical Observatory of Trieste, via G.B. Tiepolo 11, I-34143 Trieste, Italy \\ ${ }^{2}$ IFPU - Institute for Fundamental Physics of the Universe, via Beirut 2, 34151, Trieste, Italy \\ 3 INAF - Astronomical Observatory of Rome, Via Frascati 33, I-00040 Monte Porzio Catone (RM), Italy \\ ${ }^{4}$ Dipartimento di Fisica e Astronomia, Universitá di Bologna, Via Gobetti 93/2, I-40129, Bologna, Italy \\ 5 INAF - Astrophysics and Space Science Observatory of Bologna, via P. Gobetti 93/3,I-40129, Bologna, Italy \\ ${ }^{6}$ INAF - Astrophysical Observatory of Arcetri, Largo E. Fermi 5, I-50125, Firenze, Italy \\ 7 DARK, Niels Bohr Institute, University of Copenhagen, Lyngbyvej 2, DK-2100 Copenhagen, Denmark \\ ${ }^{8}$ Tianjin Astrophysics Center, Tianjin Normal University, Binshuixidao 393, 300384, Tianjin, China \\ ${ }^{10}$ Instituto de Investigación Multidisciplinar en Ciencia y Tecnología, Universidad de La Serena, Raul Bitrán 1305, La Serena, Chile \\ ${ }^{11}$ Departamento de Física y Astronomía, Universidad de La Serena, Av. Juan Cisternas 1200 Norte, La Serena, Chile \\ 12 SUPA Scottish Universities Physics Alliance, Institute for Astronomy, University of Edinburgh, Royal Observatory, Edinburgh EH9 3 HJ \\ 13 The Cosmic Dawn Center, Niels Bohr Institute, University of Copenhagen, Juliane Maries Vej 30, DK-2100 Copenhagen, Denmark \\ 14 Space Telescope Science Institute, 3700 San Martin Drive, Baltimore, MD 21218, USA \\ 15 ESO-Chile, Alonso de Cordova 3107, Vitacura, Santiago, Chile
}

Accepted ... Received ...

\begin{abstract}
In this work, we study the evolution of the mass-metallicity relations (MZRs) as predicted by the GAlaxy Evolution and Assembly (GAEA) semi-analytic model. We contrast these predictions with recent results from the VANDELS survey, that allows us to expand the accessible redshift range for the stellar MZR up to $z \sim 3.5$. We complement our study by considering the evolution of the gas-phase MZR in the same redshift range. We show that GAEA is able to reproduce the observed evolution of the $z<3.5$ gas-phase MZR and $z<0.7$ stellar MZR, while it overpredicts the stellar metallicity at $z \sim 3.5$. Furthermore, GAEA also reproduces the so-called fundamental metallicity relation (FMR) between gas-phase metallicity, stellar mass and star formation rate (SFR). In particular, the gas-phase FMR in GAEA is already in place at $z \sim 5$ and shows almost no evolution at lower redshift. GAEA predicts the existence of a stellar FMR, that is, however, characterized by a relevant redshift evolution, although its shape follows closely the gas-phase FMR. We also report additional unsolved tensions between model and data: the overall normalization of the predicted MZR agrees with observations only within $\sim 0.1$ dex; the largest discrepancies are seen at $z \sim 3.5$ where models tend to slightly overpredict observed metallicities; the slope of the predicted MZR at fixed SFR is too steep below a few $\mathrm{M}_{\odot} \mathrm{yr}^{-1}$. Finally, we provide model predictions for the evolution of the MZRs at higher redshifts, that would be useful in the context of future surveys, like those that will be performed with JWST.
\end{abstract}

Key words: galaxies: formation - galaxies: evolution - galaxies: abundances

\footnotetext{
* E-mail: fabio.fontanot@inaf.it
} 


\section{INTRODUCTION}

The observed scaling relations between galaxy properties have always been considered primary indicators to study the evolution of galaxies, and constrain the underlying physical processes. As such, the correct prediction of these relations has always been a key target for theoretical models of galaxy formation and evolution. The intrinsic complexity of the non-linear mechanisms acting on the baryonic component of the Universe prevents an accurate description of the evolution of the baryonic components of the Large Scale Structure, whose statistical properties are well recovered using numerical techniques (i.e. N-body simulations).

The study of the relative content of chemical elements in galaxies of different properties provides, in particular, tight constraints on theoretical models. Metals, i.e. chemical elements other than Hydrogen and Helium, are synthesised in the different stages of stellar evolution, corresponding to the different nuclear burning sequences that power stellar emission. As such, the stellar metallicity of a given galaxy, i.e. its content of metals relative to Hydrogen and Helium, records the effect of different physical mechanisms, primarily star formation, but also the balancing between inflows (bringing cold pristine gas into the system) and outflows (ejecting enriched gas in the surrounding environment). Moreover, the relative abundances of specific elements provide insight on the details of the star formation history, as their production depends on different stellar populations and different timescales (see Maiolino \& Mannucci 2019, for a review).

The existence of well defined scaling relations between galaxy metallicity and stellar mass (mass-metallicity relations or MZR) is therefore of great interest for understanding galaxy evolution. These scaling relations hold for both the stellar metallicity $\left(Z_{\star}\right)$ and for the metallicity of the ionized gas $\left(Z_{\mathrm{g}}\right.$, computed from the $[O / H]$ abundance ratio) in the inter-stellar medium (ISM). The Sloan Digital Sky Survey (SDSS) spectroscopic observations have provided a benchmark for the local stellar MZR (Gallazzi et al. 2005) and gas-phase MZR (Tremonti et al. 2004). Further work showed that local quiescent and star forming galaxies follow two separate MZRs (Peng et al. 2015; Trussler et al. 2020), that is usually interpreted as an evidence for "strangulation" processes (i.e. the suppression of star formation activity due to the lack of newly accreted gas onto the galaxy). As an alternative explanation, Spitoni et al. (2017) proposed that these differences in chemical composition can be explained by short gas infall timescales in the early phases of formation of present-day quiescent galaxies and by strong outflows in low-mass star forming galaxies. No clear evidence for a direct dependence of the MZR on environment has been found (Pasquali et al. 2012; Namiki et al. 2019).

The stellar MZR shows little evolution at intermediate redshifts $z \lesssim 1$ (e.g. Ferreras et al. 2009; Gallazzi et al. 2014), but a relevant evolution at higher redshifts Sommariva et al. (2012); Cullen et al. (2019). A more significant evolution has been found for the gas-phase MZR at $1.5<z<3.5$ (Erb et al. 2006; Maiolino et al. 2008; Pérez-Montero et al. 2013; Zahid et al. 2014b; Troncoso et al. 2014; Steidel et al. 2014; Onodera et al. 2016; Curti et al. 2020b). At all redshifts, the MZR shows a similar shape, with increasing metallicity at increasing stellar mass, and an overall metallicity decrease at increasing redshift. However, the differential evolution is characterized by the so-called downsizing in metallicity (Maiolino et al. 2008; Fontanot et al. 2009): lowmass galaxies exhibit a larger metallicity evolution from $z \sim 3.5$ to $z \sim 0$ than their more massive counterparts.

Already Tremonti et al. (2004) studied secondary de- pendences in the MZR; later Mannucci et al. (2010, see also Lara-López et al. 2010; Hunt et al. 2012) proposed a fundamental metallicity relation (FMR) between gas-phase metallicity, stellar mass and star formation rate (SFR). Further studies at higher redshift suggest a negligible evolution of the FMR up to $z \sim 2.5$ (Cresci et al. 2012, 2019; Sanders et al. 2020; Curti et al. 2020b). This stability of the FMR is usually interpreted in the framework of equilibrium models in which gas infall is balanced by star formation and feedback-driven outflows (see e.g. Dayal et al. 2013). The observed MZRs thus represent projections of the FMR, and the observed redshift evolution is due to different regions of the FMR being sampled at different redshifts, because of the evolution of the cosmic star formation rate. More recent works find that a stronger secondary dependence is measured with the gas content rather than SFR (Bothwell et al. 2013), and that the SFR-defined FMR is a projection of this more fundamental relation (Bothwell et al. 2016; Brown et al. 2018). The accepted interpretation of available data is that the role played by outflows as SFR regulators is minimal, and that the shape and evolution of the FMR is mainly driven by the cosmological evolution of the cold gas content. A discussion about the role of gas (and more precisely the gas-to-stellar mass ratio) can be found in Zahid et al. (2014a) and Curti et al. (2020b), who suggest that the evolution of the MZR and FMR is driven by the evolution of the gas/star mass ratio. Indeed, Ellison et al. (2008) suggest that the MZR sensitivity to SFR efficiency is the most likely origin for the trends they report as a function of specific star formation $\left(\mathrm{sSFR}=S F R / \mathrm{M}_{\odot}\right)$ and galaxy size.

Despite a large amount of focused work, these studies are still potentially affected by significant biases, mostly due to the different techniques used to derive metallicities from spectroscopic observations and to uncertainties on the relative calibrations (see Kewley \& Ellison 2008; Maiolino \& Mannucci 2019, for an extensive reviews). Stellar metallicity estimates mostly rely on the comparison between stellar absorption features with distinct sensitivity to age and metallicity (e.g. the Lick indices) and the predictions from Stellar Population Synthesis (SPS) models. The derived estimates depend on the SPS models used, on the features or spectral range analysed, and on the assumptions made for the galaxy star formation history. Spectral features in the Ultra-Violet (UV) are also used to estimate the stellar metallicity, mostly in high-redshift galaxies, of the younger stellar component. Gas metallicities are usually based on the analysis of strong emission line ratios, that could be calibrated in two different ways. The so-called direct approach (see e.g. Pilyugin et al. 2010; Pérez-Montero 2014) relies on measuring the temperature of the different ionization zones. Alternatively photoionization models are used to generate a grid of synthetic spectra to be compared with observations.

It is important to keep in mind that most of these approaches need an absolute calibration that is usually obtained by comparison with theoretical models of stellar evolution. Moreover, HII regions in galaxies are expected to cover a wide range of physical properties and direct methods can only give an estimate of the mean emission. These considerations imply that the accuracy of the measurements is limited by our knowledge of the physics of ionized regions and/or stellar evolution and relies on a number of assumptions to break the complex degeneracy between age and metallicity. These considerations explain the apparent tensions between results based on different methods. Photoionization models tend to overestimate the gas-phase metallicity by 0.2-0.6 dex (Kewley \& Ellison 2008), while the direct methods may differ by up to a factor of a few (Tsamis et al. 2003). Generally, direct measurement using UV 
spectra provide a good match of the metallicities of young stellar population, although they might be biased toward lower metallicities due to the presence of temperature fluctuations (Bresolin et al. 2016; Curti et al. 2020a).

Theoretical models of galaxy evolution have long been reported to face tensions in reproducing the observed MZRs (Somerville \& Davé 2015), and in particular their redshift evolution. These discrepancies have been reduced with the new generation of models (Hirschmann et al. 2016; Xie et al. 2017; Lagos et al. 2016; Torrey et al. 2019): these models also provide a better overall description of the assembly history of the galaxy populations, solving long standing issues with the evolution of lowmass galaxies (both semi-analytic - Fontanot et al. 2009 - and numerical simulations Weinmann et al. 2012) and the local fraction of quenched galaxies (De Lucia et al. 2019; Xie et al. 2020).

In a recent series of papers, we introduce the GAlaxy EVolution and Assembly (GAEA) model. This model is able to correctly reproduce the evolution of the GSMF and cosmic SFR up to the highest redshift accessible (Fontanot et al. 2017). The same model is also able to reproduce both the stellar and gas-phase $z \sim$ 0 MZRs (Hirschmann et al. 2016). In particular, De Lucia et al. (2020) study the scatter around the $z \sim 0$ gas-phase MZR, showing that it is primarily regulated by the cold gas inflow/accretion rate on the model galaxies. In this paper we now focus on the redshift evolution of the MZRs and FMR as predicted by GAEA, going beyond the preliminary comparison shown in (Hirschmann et al. 2016).

This paper is organised as follows. In Section 2 and 3 we will present, respectively, our semi-analytic model of galaxy formation and the datasets we will consider for the comparison with the theoretical predictions shown in Section 4. We will then discuss our conclusions in Section 5. Finally, we will summarise our conclusions in Section 6.

\section{SEMI-ANALYTIC MODEL}

In this work we consider predictions from the GAlaxy Evolution and Assembly (GAEA) semi-analytic model (SAMs). SAMs represent a theoretical tool to predict the evolution of galaxy populations across cosmological epochs and volumes. They assume that galaxies form inside dark matter haloes from the condensation of gas. A complex network of physical processes is then responsible for the cooling and heating of the gas, as well as for the matter and energy exchanges between the different gas phases and galaxy components (disc, bulge and halo). In order to amend for our limited knowledge of the details of the relevant mechanisms, SAMs are defined by using a system of differential equations based on parametrizations constructed either on empirical results or theoretical arguments. This approach translates into a limited computational request (when compared, e.g., with hydrodynamical simulations) and thus allows a characterization of the role of individual mechanisms in shaping galaxy properties.

GAEA is especially well suited for the comparison presented in this paper, thanks to its improved modelling of stellar feedback (Hirschmann et al. 2016). This leads to a prediction for the evolution of the cosmic star formation rate and galaxy stellar mass function in good agreement with observational constraints up to the highest redshifts available (Fontanot et al. 2017). In particular, GAEA is able to reproduce the evolution of the low-mass end of the galaxy stellar mass function at $z<3$, that has represented a long standing problem for models of galaxy evolution (Fontanot et al. 2009; Weinmann et al. 2012). This success is due to the implemen- tation of an ejective feedback scheme that combines (a) the numerical results for gas reheating from Muratov et al. (2015), (b) the outflow rate description from Guo et al. (2011) and (c) the gas reincorporation analysis from Henriques et al. (2013). Given the correct distribution of stellar masses at different cosmic epochs in GAEA, checking the chemical enrichment levels is thus an interesting test for this SAM. We already show in Hirschmann et al. (2016) that GAEA is able to reproduce the evolution of the MZRs up to $z \sim 2$. In this paper, we will deepen this analysis using the most recent results covering the redshift range $0.7<z<3.5$ both for the stellar and gas-phase MZRs and FMR.

GAEA also includes an advanced treatment for chemical enrichment (De Lucia et al. 2014), that allows us to trace the evolution of the abundances of different metal species. This scheme accounts for the different lifetime of stars of different initial mass (Padovani \& Matteucci 1993) and traces their individual contribution to chemical enrichment, via differential yields. In detail, we assume the following yields: (a) for the lowest mass stars contributing to the chemical enrichment of the ISM (i.e. $m_{\star}<8 \mathrm{M}_{\odot}$, that end their lives as asymptotic giant branch stars) we consider the Karakas (2010) yields; (b) more massive stars are expected to end their evolution as SNeII, whose yields are taken from Chieffi \& Limongi (2002); (c) finally for SNeIa we use yields from Thielemann et al. (2003). These assumptions imply that the global metal enrichment is a direct GAEA prediction and it is not regulated by a free parameter as is typically the case when adopting an instantaneous recycling approximation.

In this paper, we use a sample including all model galaxies (i.e. both centrals and satellites) extracted from our reference GAEA realization run on the Millennium Simulation $\left(\Omega_{\Lambda}=0.75\right.$, $\Omega_{m}=0.25, \Omega_{b}=0.045, n=1, \sigma_{8}=0.9, H_{0}=73 \mathrm{~km} / \mathrm{s} / \mathrm{Mpc}$ Springel et al. 2005). Only for the gas-phase MZR, we restrict the sample to model galaxies with a meaningful gas fraction (e.g. $f_{\text {gas }}=M_{\text {gas }} / M_{\star}>0.1$ ), as the observational samples used for comparison are typically composed of star-forming/gas-rich galaxies. Moreover, this selection removes model galaxies with unrealistic metallicity levels due to the dearth of gas. We check that using other selection criteria, such as restricting the sample to either central galaxies ${ }^{1}$, or actively star-forming galaxies (e.g. using a standard $s S F R>0.3 / t_{\text {hubble }}$ threshold), does not change our conclusions qualitatively. In all plots we show the median MZR and FMR, and the corresponding 15th-85th percentile range.

In the following, gas-phase metallicities have been estimated from the predicted mass fraction of the oxygen element in the coldgas phase of the model galaxy. Stellar metallicities have been computed by dividing the mass in metals locked into long-lived stars by the total stellar mass of the model galaxy, that represents the intrinsic metallicity of the stellar component in GAEA. This estimate could differ from observational estimates used in this work which are based on either optical absorption features (tracing both Fe and $\alpha$-elements, weighing more the old stellar populations) or the properties of the FUV spectral range (tracing mostly Fe, weighing more dusty young stellar populations). As a general example, we quote the results discussed in Nelson et al. (2018), warning the reader that these cannot be extrapolated to all theoretical models of galaxy evolution. These authors compare the intrinsic stellar metallicities

1 The evolution of satellite galaxies in GAEA depends on the treatment of environmental processes (see e.g. De Lucia et al. 2019). In the H16F model, in particular, satellites are subject to instantaneous removal of their hot gas (i.e. "strangulation"), which affects heavily their subsequent evolution. 
in the ILlustrisTNG simulation (Pillepich et al. 2018), with the corresponding quantities derived from synthetic mock spectra build using ILLUSTRISTNG metallicity and star formation histories. For their model galaxis, they find that spectroscopically derived median $Z_{\star}$ are offset low by $\sim 0.2-0.5$ dex at $M_{\star}<10^{10.5} M_{\odot}$, with respect to intrinsic metallicities.

\section{DATASET}

We consider stellar metallicity measurements from the VANDELS ESO public spectroscopic survey (McLure et al. 2018; Pentericci et al. 2018) carried out with the VIMOS spectrograph on ESO's Very Large Telescope (VLT). The targeted fields are centred on the CANDELS CDFS and UDS, that already include extensive WFC3/IR imaging (Grogin et al. 2011; Koekemoer et al. 2011) and for which photometric catalogues are available (see e.g. Guo et al. 2013). The main targets of the VANDELS survey are (a) massive passive galaxies at $1<z<2.5$, (b) bright star-forming galaxies at $2.4<z<5.5$ and (c) faint star-forming galaxies at $3<z<7$. Pentericci et al. (2018) provides more details on the observations and reduction techniques. Stellar masses are derived from SED fitting as described in McLure et al. (2018).

We take advantage of the results obtained by Cullen et al. (2019) and Calabrò et al. (2021) for the stellar MZR at $z \sim 3.5$. Cullen et al. (2019) consider a sample of 681 star forming galaxies at $2.5 \lesssim z \lesssim 5$ from the second VANDELS data release with robust redshift determination. Since the typical S/N of VANDELS spectra is not high enough to extract informations from individual sources, stacked spectra in stellar mass and redshift are used. Metallicities are then derived from full UV spectral fitting including indicators that trace the stellar photospheric metallicity of young stellar populations. Recently, Calabrò et al. (2021) propose an alternative analysis for stellar metallicities in the most recent VANDELS sample of 732 star-forming galaxies, that complements the Cullen et al. (2019) study. In particular, Calabrò et al. (2021) adopt different measurements of stellar metallicity from individual UV absorption features. They consider stacked spectra and restframe UV spectral features ( $\lambda 1501$ and $\lambda 1719)$. The two estimates are in good agreement: in particular they share a similar dependence on stellar mass, increasing $\sim 0.5$ dex from $M_{\star} \sim 10^{9} \mathrm{M}_{\odot}$ to $M_{\star} \sim 10^{10.5} \mathrm{M}_{\odot}$. In order to put these results into a wider context, we also consider results on the stellar phase metallicity coming from other surveys at lower redshifts, such as Zahid et al. (2017) and Gallazzi et al. (2014). These studies are based on stellar metallicities derived from optical spectral features, instead of the UV features used in the VANDELS analysis. Optical features are sensitive to older stellar populations than UV features. Furthermore, for consistency with the VANDELS sample considered in this paper, we refer only to results obtained from star-forming subsamples in Zahid et al. (2017) and Gallazzi et al. (2014).

In order to provide a more comprehensive picture of galaxy formation, we consider not only the stellar MZR, but also the gas-phase MZR and its redshift evolution. We thus complement VANDELS data with recent results from the KMOS Lensed Emission Lines and VElocity Review (KLEVER Curti et al. 2020b). KLEVER employs the multi-object near-IR integral field spectrograph KMOS on VLT. It focuses on the resolved properties of 39 galaxies in the redshift range $1.2<z<2.5$. In detail, the spectral resolution of the KMOS instrument allows an accurate mapping of bright nebular emission lines at rest-frame, including $\mathrm{H}_{\alpha}$,

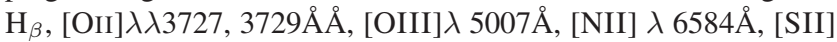

$\lambda \lambda 6717,31 \AA \AA \AA$, [SIII] $\lambda \lambda 9068,9530 \AA \AA$, fundamental for the estimate of the metallicity of the cold gas. Stellar masses for the KLEVER sources have been derived from SED fitting, while their SFRs are computed from the estimated intrinsic $\mathrm{H}_{\alpha}$ luminosity. In order to have an homogeneous sample of gas-phase determinations, Cresci et al. (2019) collect gas-phase metallicities from several authors (see caption of Fig. 1 for all references) and convert all stellar masses and SFRs determinations to the same Chabrier IMF. More importantly, they re-evaluate individual gas-phase metallicities using the strong-line (e.g. $\mathrm{N}_{\mathrm{II}} / \mathrm{H}_{\alpha}$ ) calibration as in Curti et al. (2020b) for all samples.

\section{RESULTS}

\subsection{Mass-Metallicity relations}

We first consider the evolution of the mass-metallicity relations (MZRs). In the left panel of Fig. 1 we compare the evolution of the stellar MZR in the redshift range $2.5 \lesssim z \lesssim 5$ with data from VANDELS (Cullen et al. 2019; Calabrò et al. 2021; Talia et al. 2021), and with data at lower redshifts from the literature (Gallazzi et al. 2014; Zahid et al. 2017). All data have been rescaled to a common 0.02 solar metallicity. GAEA predictions reproduce reasonably well the local and the $z \sim 0.7$ relations. The model predicts an evolution of the MZR normalization to $z \sim 3$ of roughly $\sim 0.35$ dex in metallicity. This is roughly $\sim 0.25$ dex less than the evolution suggested by the Cullen et al. (2019) and Calabrò et al. (2021). We stress however, that the tension between model predictions and the highest-redshift data available is at $1-\sigma$ level. It is important to keep in mind that all observational results at this redshift are based on the VANDELS dataset, but they differ both in terms of sample size and analysis strategy. Nonetheless, all VANDELS measurements rely on spectral features in the FUV spectral range. It is expected that FUV-weighted metallicities could be offset low by $\sim 0.1$ dex with respect to mass-weighted metallicities (see e.g. Cullen et al. 2019). This is due to the fact that the youngest stellar populations, that dominate the UV flux, are at the same time the most metal rich and the most dust obscured. Moreover, FUV-weighted metallicities mainly trace the iron abundance, as a proxy to total metallicity, and this can also contribute to the offset from model predictions. More interestingly, sources included in the (Cullen et al. 2019) sample have a median sSFR of about $10^{-8.35} \mathrm{yr}^{-1}$, that is quite different from the typical sSFR $\sim 10^{-8.7} \mathrm{yr}^{-1}$ for $\mathrm{z} \sim 3.5$ galaxies in GAEA. We will deepen this point in Section 5. Overall, the VANDELS stellar MZR shows a steeper slope than the stellar MZR predicted by GAEA. Calabrò et al. (2021) study in detail the effect of VANDELS selection criteria on the resulting slope of the $z \sim 3.5 \mathrm{MZR}$ and show that when the same criteria are applied on GAEA mock catalogues the predicted MZR slope is in better agreement with the observed one (although the discrepancy in the normalization holds). As in this paper we are mainly concerned about the predicted redshift evolution of the global MZRs, we do not consider selection effects while dealing with individual datasets.

We then consider the evolution of the gas metallicity as a function of the stellar mass in the right panel of Fig. 1. We compare GAEA predictions with a variety of data from several surveys as indicated in the legend. We remind the reader that in this paper we are considering the homogeneized values from Cresci et al. (2019, filled symbols), i.e. after reporting all individual datasets to the same calibration framework used in Curti et al. (2020b, see previous section for more details). The advantage of such a large compilation of homogeneized data lies in the possibility of studying 

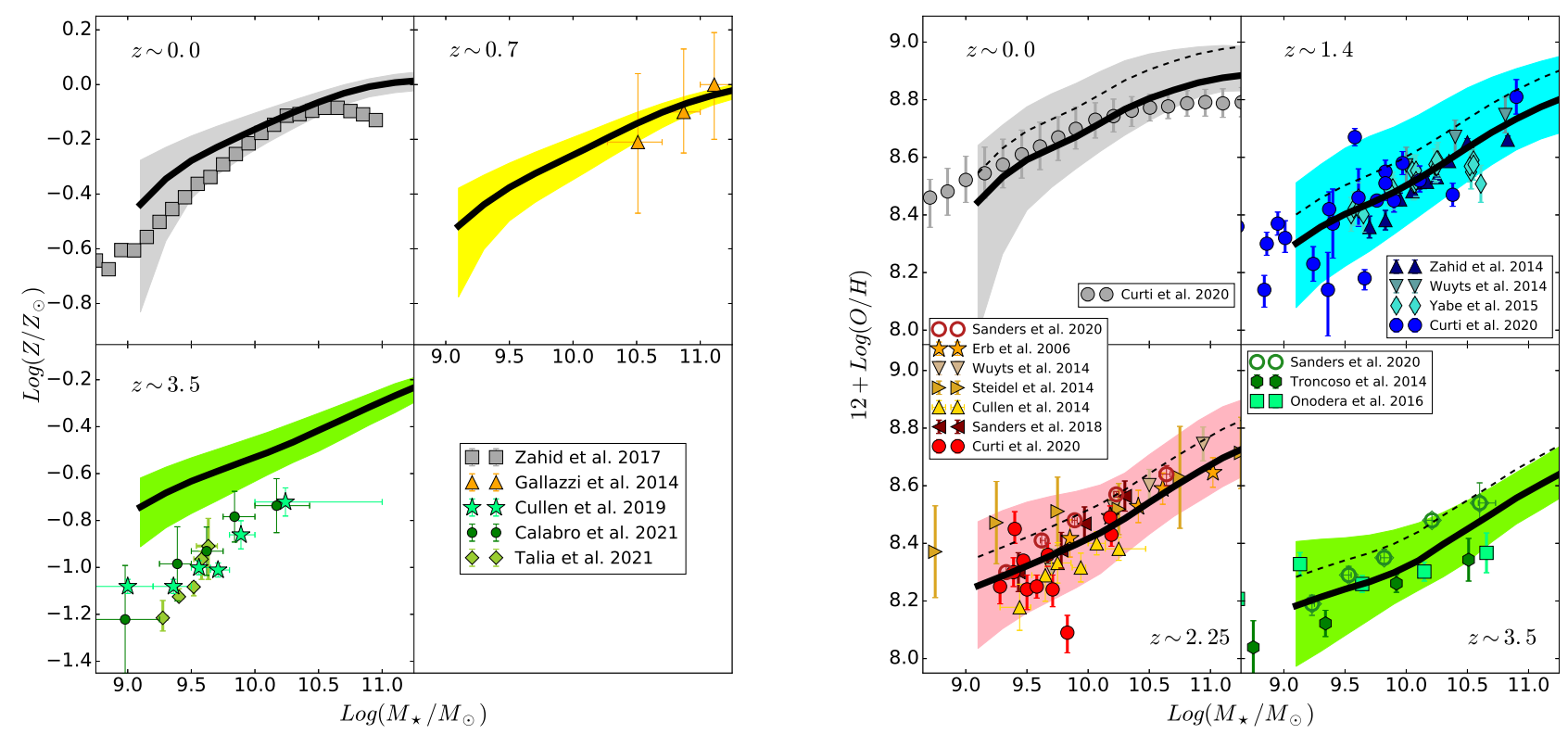

Figure 1. Left panel: Redshift evolution of the stellar MZR relation. Datapoints with errorbars refer to the estimates from Zahid et al. (2017, grey squares), Gallazzi et al. (2014, dark yellow triangles), Cullen et al. (2019, pale green stars), Calabrò et al. (2021, green circles) and Talia et al. (2021, olive diamonds). All data have been rescaled to a common 0.02 solar metallicity. Right panel: Redshift evolution of the cold gas MZR. Datapoints with errorbars refer to the estimates from Curti et al. (2020a, grey circles), Wuyts et al. (2014, light blue and pale brown triangles), Zahid et al. (2014b, dark blue triangles), Yabe et al. (2015, cyan diamonds), Curti et al. (2020b, blue and red circles), Erb et al. (2006, orange stars), Steidel et al. (2014, orange triangles), Cullen et al. (2014, gold triangles), Sanders et al. (2018, brown triangles), Troncoso et al. (2014, green triangles) and Sanders et al. (2020, open circles). Solid symbols show data that have been rescaled to the same assumptions as in Curti et al. (2020b). In all panels, the thick black solid lines represent the corresponding median MZRs as predicted by GAEA at the appropriate redshift, with the shaded area referring to the 15th-85th percentiles. Stellar phase MZRs are drawn from a model sample including all model galaxies, while gas-phase from a model sample including only gas rich galaxies. In the right panel, solid black lines refer to GAEA predictions shifted 0.1 dex downwards, while the thin dashed lines represent the intrinsic model predictions (see text for more details).

in detail the absolute evolution of the MZR, under the hyphothesis that all data suffer from similar systematics at all redshift (see also Maiolino et al. 2008). Moreover, the still relevant calibration uncertainties between the several spectral indicators adopted in the literature limit the constraining power of single epoch MZRs to the shape of the relation. Therefore, the relative evolution of the metal enrichment at fixed stellar mass at different cosmic epochs represents a more fundamental test for models of galaxy evolution. It is worth stressing that in Hirschmann et al. (2016), we compared GAEA predictions with the Tremonti et al. (2004) data for the $z \sim 0$ gas-phase MZR, that are systematically higher than Curti et al. (2020a). In De Lucia et al. (2020), we compare GAEA predictions with both Tremonti et al. (2004) and Curti et al. (2020a) measurements. GAEA intrinsic predictions are indeed systematically higher than the collection by Cresci et al. (2019) (thin dashed lines in Fig. 1, right panel). In order to focus on the redshift evolution of the MZR it is therefore useful to shift downwards GAEA predictions by $0.1 \mathrm{dex}$, based on the match to the $z \sim 0$ data. It is important to stress, that this shift does not correspond to any retuning of the model parameters with respect to Hirschmann et al. (2016). Fig. 1 clearly shows that, under this hypothesis, GAEA is able to recover the overall evolution of the gas-phase MZR up the highest redshifts available and the predicted scatter is also compatible with the available datasets, although some tensions with the predicted slope of the relations hold (e.g. at $\mathrm{z}=0$ it is somewhat steeper than observed, De Lucia et al. 2020). It is therefore important to stress that our results do not imply that a constant shift of the predictions by the same amount is enough to account for all the systematics in the observed data. In particular, we do not expect these systematics to be the same at all galaxy mass scales, but they should depend also on the mass scale under scrutiny and affect the overall slope of the relation as well (as a matter of fact the predicted slopes do not match perfectly the observed determinations even after renormalization). Nonetheless, we deemed that our approach allows us to explore if GAEA is able to predict the same evolutionary trends in the observed data (irrespective of the true overall normalization), both as a function of redshift and stellar mass. Moreover, the 0.1 dex shift is acceptetable, given the expected uncertainty in the normalization of the MZR, as due to the calibration of the spectral indicators used to derive gas-phase metallicities and to the set of reference theoretical templates (Kewley \& Ellison 2008). In particular, Curti et al. (2020b) argue that the electron temperature method could suffer from a systematic underestimate of $0.1 \mathrm{dex}$, due to the presence of temperature fluctuations in the HII regions. Therefore, we conclude that GAEA is able to reasonably reproduce the observed evolution rate in both stellar and gas-phase metallicity from $z \sim 3-3.5$ to $z \sim 0$, that is a more stringent constraint for galaxy evolution than the overall normalization of the relation, that may also depend on the assumption made for the metallicity estimate (Kewley \& Ellison 2008).

While working on this paper, Sanders et al. (2020) published new results on the gas-phase MZR in the range $2.3<z<3.3$, obtained in the context of the MOSDEF survey, using the MultiObject Spectrometer For Infrared Exploration (MOSFIRE) instru- 

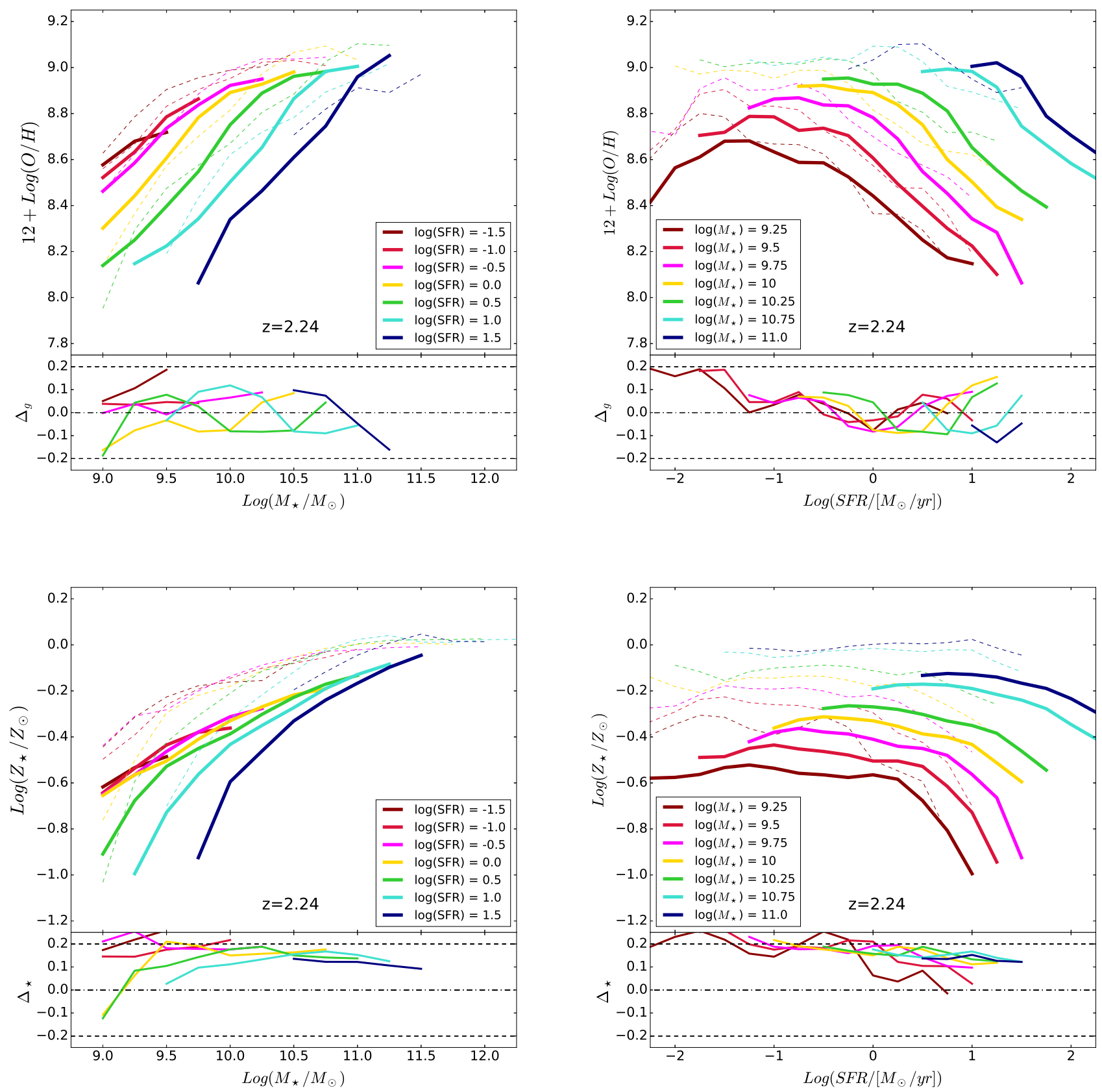

Figure 2. Fundamental Metallicity Relation between SFR, $M_{\star}$ and cold gas metallicity at $z \sim 2.25$ in GAEA. Upper left panel: the cold gas metallicity - stellar mass relation predicted in GAEA in different bins of $\log (S F R)$. Upper right panel: the cold gas metallicity - SFR relation predicted in GAEA in different bins of $\log \left(M_{\star}\right)$. Lower left panel: stellar mass-metallicity relation predicted in GAEA in different bins of $\log (S F R)$. Lower right panel: the $Z_{\star}$ - SFR relation predicted in GAEA in different bins of $\log \left(M_{\star}\right)$. In all four panels, thin dashed lines show the corresponding relations at $z=0$ and the lower insets report the differences $\Delta_{g}=\log \left(Z_{\text {gas }}(z)\right)-\log \left(Z_{\text {gas }}(0)\right)$ and $\Delta_{\star}=\log \left(Z_{\star}(z)\right)-\log \left(Z_{\star}(0)\right)$.

ment. These metallicity determinations are based on the analysis of spectral features on stacked spectra; however it has not been possible to repeat the homogeneization procedure discussed previously. For this reason, we show these results as open circles in Fig. 1: it is worth noting that the MZR relation they trace agrees quite well with the GAEA intrinsic relation (dashed lines). This confirms both the intrinsic dispersion of the MZRs based on different calibrations, and the fact that GAEA is able to reproduce the redshift evolution of the MZR, as constrained by homoegeneous MZR determinations.

\subsection{Fundamental Metallicity Relation}

We then consider in detail the relation between the cold gas metallicity, the SFR and $M_{\star}$. Mannucci et al. (2010) first showed that the mass-metallicity relations we discussed in previous paragraphs 


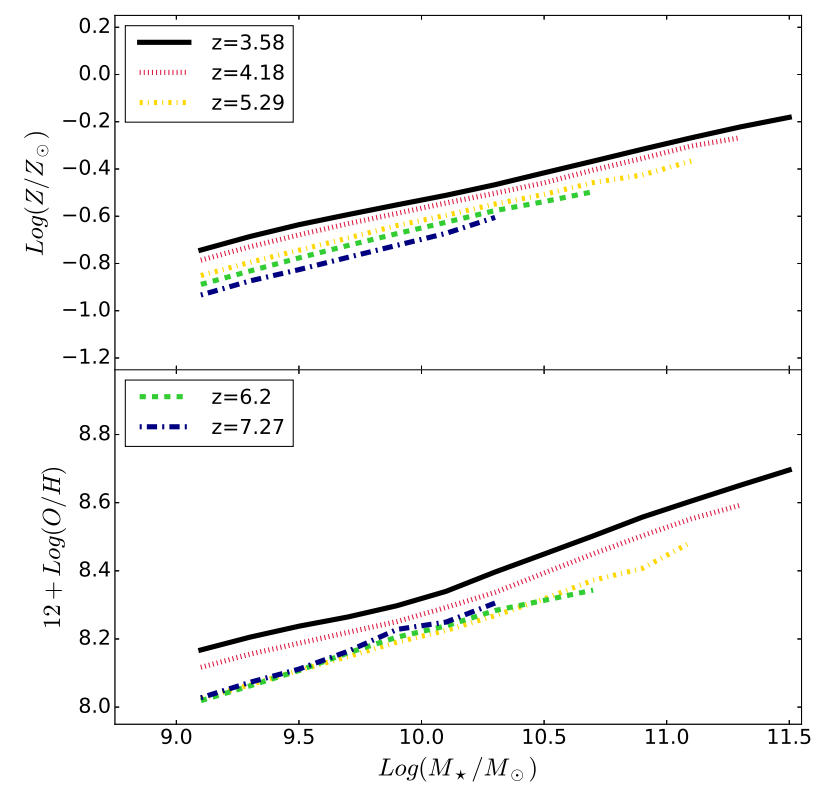

Figure 3. Predicted evolution of the stellar (upper panel) and gas-phase (lower panel) MZR at $3.5 \lesssim z \lesssim 7.2$.

are just a projection of a more general relation between $M_{\star}$, SFR and cold gas metallicity (Fundamental Metallicity Relation, FMR). Moreover, Mannucci et al. (2010) also show that the FMR is remarkably similar up to $\mathrm{z}=2.5$, while the limited amount of data available at higher redshifts points towards a significant evolution (Mannucci et al. 2009; Troncoso et al. 2014). Indeed, almost all $z \lesssim 2-3$ galaxies with accurate measurements of these properties lie within 0.6-0.2 dex from the $z \sim 0$ FMR. Therefore, the observed evolution in the mass-metallicity relations can be explained simply by assuming that galaxies at different redshift sample different regions of the FMR.

A detailed comparison between GAEA predictions and the $z=0$ FMR (including Curti et al. 2020a) has been presented in De Lucia et al. $(2020)^{2}$ : overall, the model predicts a well defined FMR in reasonable agreement with observations. There are, however, some evident discrepancies between predictions and data: the most notable is the different metallicity normalization (as we already mention in the previous section). Moreover, the predicted slope of the $M_{\star}-Z_{\text {gas }}$ relation at fixed $S F R$ is much steeper than observed for $S F R$ lower than a few $\mathrm{M}_{\odot} \mathrm{yr}^{-1}$. The predicted slope at the highest SFRs is in better agreement with the observational constraints.

In this paper we focus on the redshift evolution of the FMR

2 It is worth noting that in De Lucia et al. (2020) we use a model version based on predictions from the more recent version of the model from Xie et al. (2017), featuring prescriptions for splitting the cold gas phase into its $\mathrm{HI}$ and $\mathrm{H}_{2}$ components derived from Blitz \& Rosolowsky (2006). The predictions presented here, on the other hand, derive from the model implementing the "FIRE" feedback prescription presented in (Hirschmann et al. 2016): this represents our standard realization, which we show is able to provide a good agreement with the evolution of the GSMF and cosmic SFR up to $z \sim 7$ (Fontanot et al. 2017). We stress that the main trends we discuss in this paper do not depend on the specific version of the model adopted.
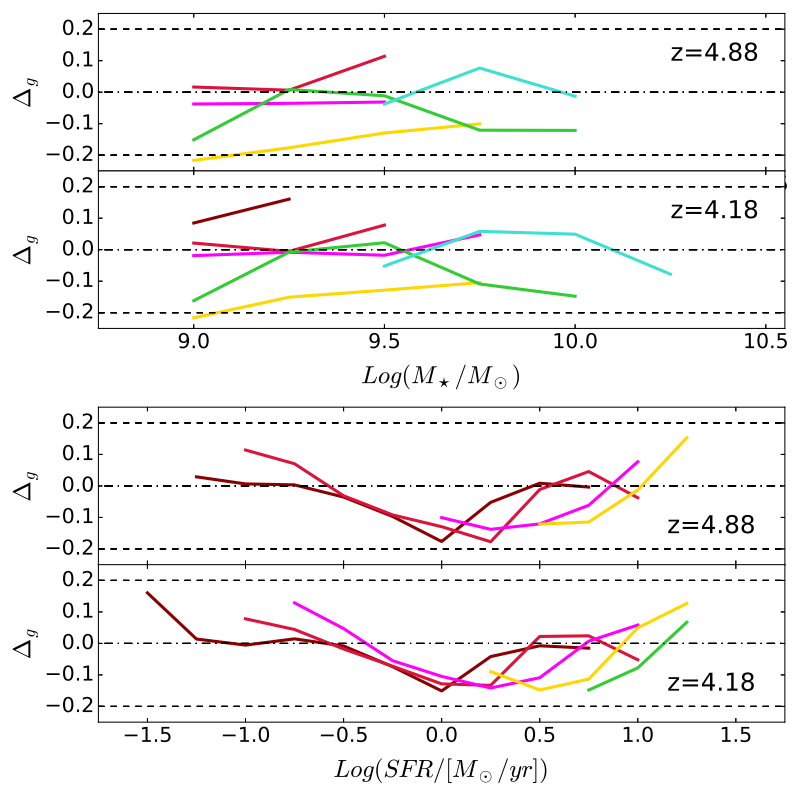

Figure 4. Evolution of the gas-phase FMR in the redshift range $z \sim 4-5$. The various panels show the evolution with respect to the $z=0$ FMR using the $\Delta_{g}=\log \left(Z_{\text {gas }}(z)\right)-\log \left(Z_{\text {gas }}(0)\right)$ indicator as in the lower insets in Fig. 2, and using the same colour scheme.

as predicted by GAEA. In the upper panels of Fig. 2 we show the FMR predicted by GAEA at $z \sim 2.24$ (solid lines). We chose this redshift as a reference since this is representative of the shape of the relation in the $1.5<z<3.5$ range. The left panel shows the $Z_{\text {gas }}-M_{\star}$ relations at fixed SFR, while the right panel shows the $Z_{\text {gas }}$-SFR relations at fixed $M_{\star}$. We then compare the shape of the distributions with the corresponding predictions at $z=0$ (thin dashed lines in all panels).

In detail we consider in the lower insert of each panel the residuals with respect to the $z=0$ FMR:

$$
\Delta_{\mathrm{g}}=\log \left(Z_{\mathrm{gas}}(z)\right)-\log \left(Z_{\mathrm{gas}}(0)\right) .
$$

where $Z_{\text {gas }}(z)$ and $Z_{\text {gas }}(0)$ represent the GAEA predicted MZRs at the considered redshift and at $z=0$. For both projections the deviation from the $z=0$ relations is smaller than $0.1-0.2 \mathrm{dex}$, in agreement with the observational evidence from Mannucci et al. (2010) and Curti et al. (2020b) for high redshift galaxies. We confirm that model galaxies populate a well defined FMR already at intermediate redshifts, and, in particular, the $z=0$ relations is already in place at $z \sim 2$.

We then consider another possible FMR, defined by using the stellar metallicity (lower panels of Fig. 2). A stellar FMR is well defined at all redshift in GAEA, with a shape similar to the gas-phase FMR. However, while the gas-phase FMR is characterized by a defined shape across all redshifts in the $M_{\star}-Z_{\star}$-SFR parameter space, the stellar FMR is characterized by a marked evolution of its overall normalization with redshift. Nonetheless, in the following we will refer to a "stellar FMR" in order to highlight its analogy with the original gas-phase FMR, but keeping in mind the different nature of this redshift-dependent relation. In particular, from $z \sim 2.25$ to $z=0$, the shift is already of the order of $\sim 0.2$ dex in metallicity 

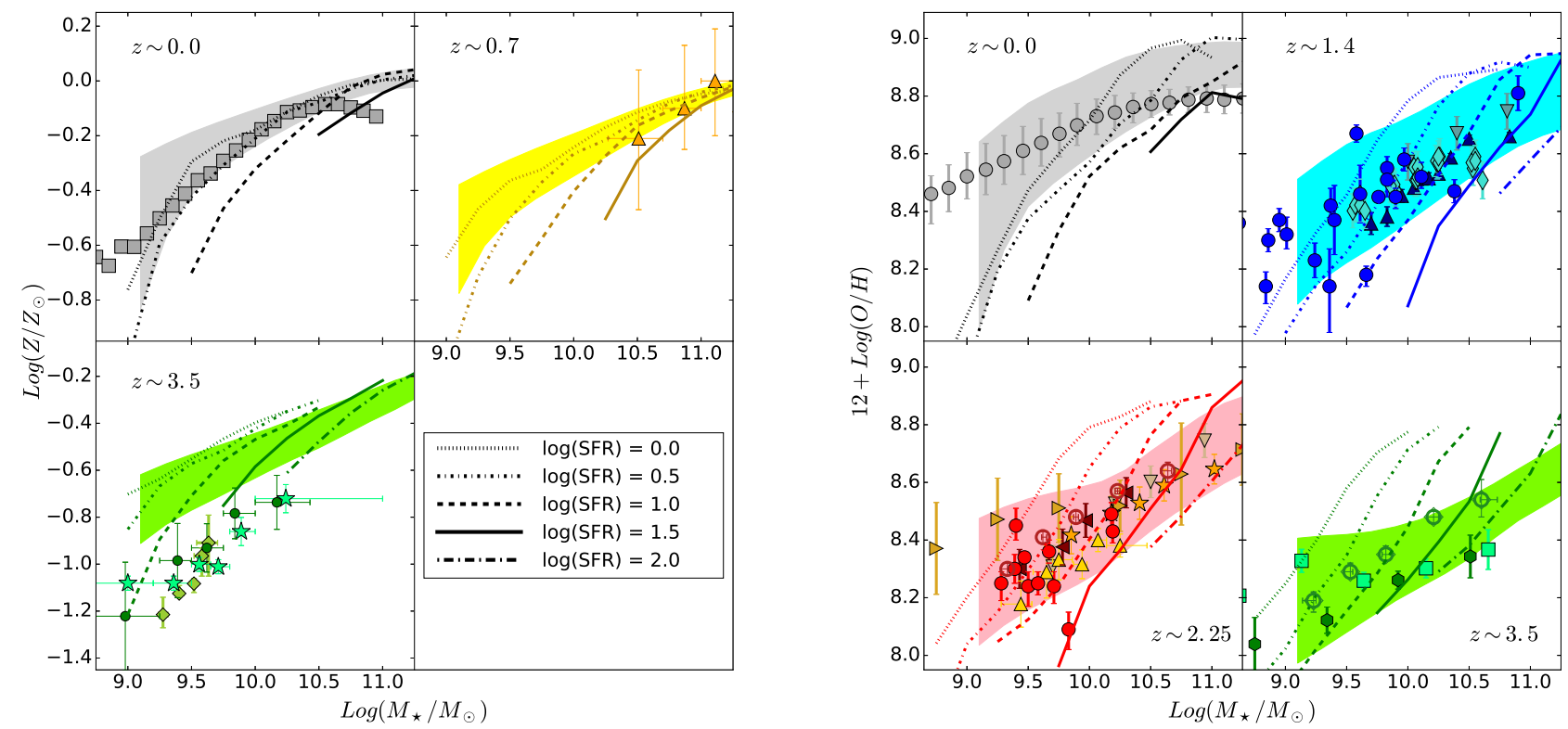

Figure 5. Stellar (left panels) and gas-phase (right panels) MZRs for samples of galaxies at a given SFR level (as indicated in the legend). All other datapoints and shaded areas as in Fig. 1.
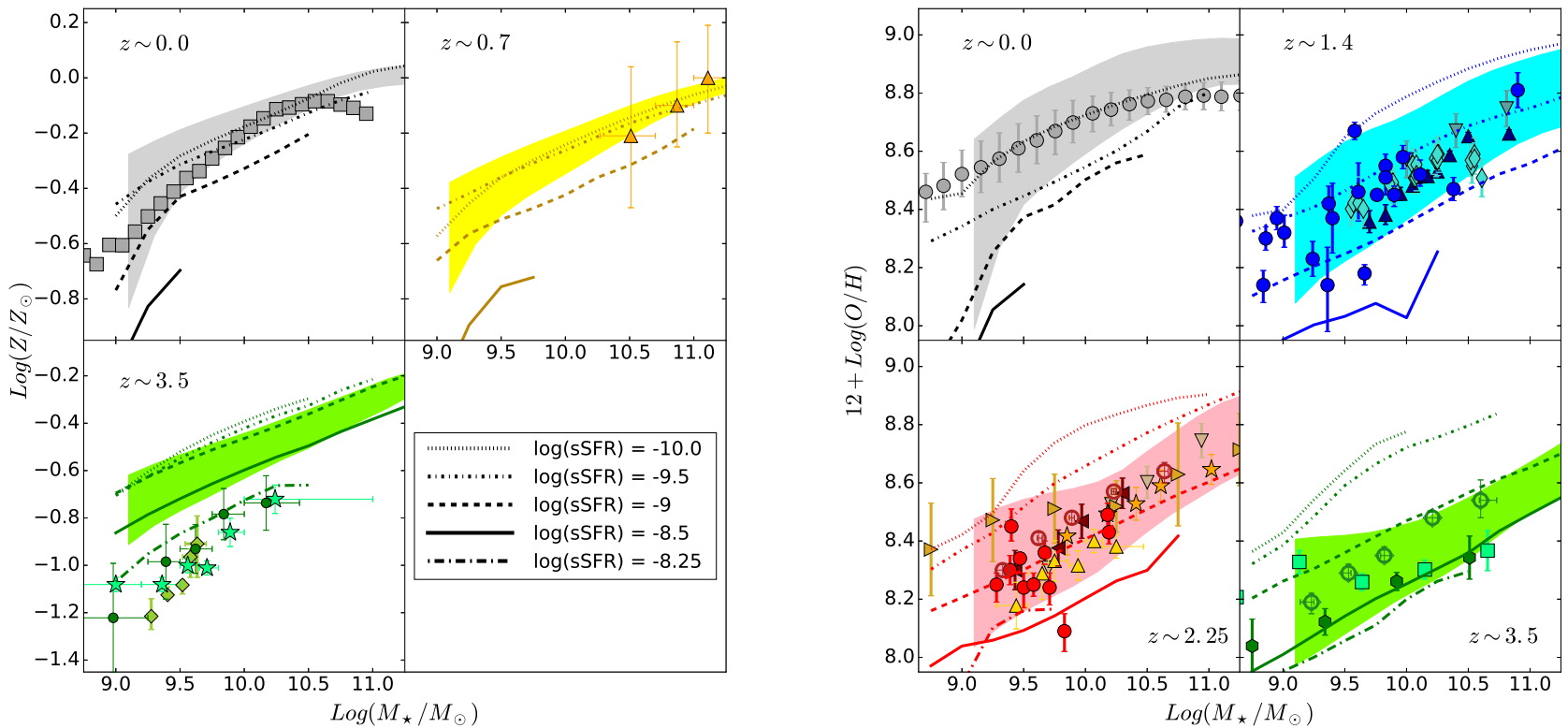

Figure 6. Stellar (left panels) and gas-phase (right panels) MZRs for samples of galaxies at a given sSFR level (as indicated in the legend). All other datapoints and shaded areas as in Fig. 1.

(we define $\Delta_{\star}$ analogously to $\Delta_{g}$ ), and keeps increasing at higher redshift (reaching $\sim 0.3-0.4$ at $\mathrm{z} \sim 5$ ). These results thus suggest that GAEA predicts a universal gas-phase FMR. On the other hand, the stellar FMR traces the increasing baryonic mass locked in the metal component as galaxies evolve.

GAEA allows us to provide predictions for the FMR at even higher redshifts, that will be possible to test thanks to future facil- ities like the James Webb Space Telescope (JWST). In particular, JWST is the only instrument that will be able to access the strong lines needed for measuring gas-phase metallicities beyond $z \sim 3.5$. Fig. 3 shows the expected evolution of the total stellar and gasphase MZR at $3.5 \lesssim z \lesssim 7.2$. The stellar MZR is characterized by a steady evolution of the normalization and a constant slope, while the gas-phase MZR seems to evolve only up to $z \sim 5$. For both re- 

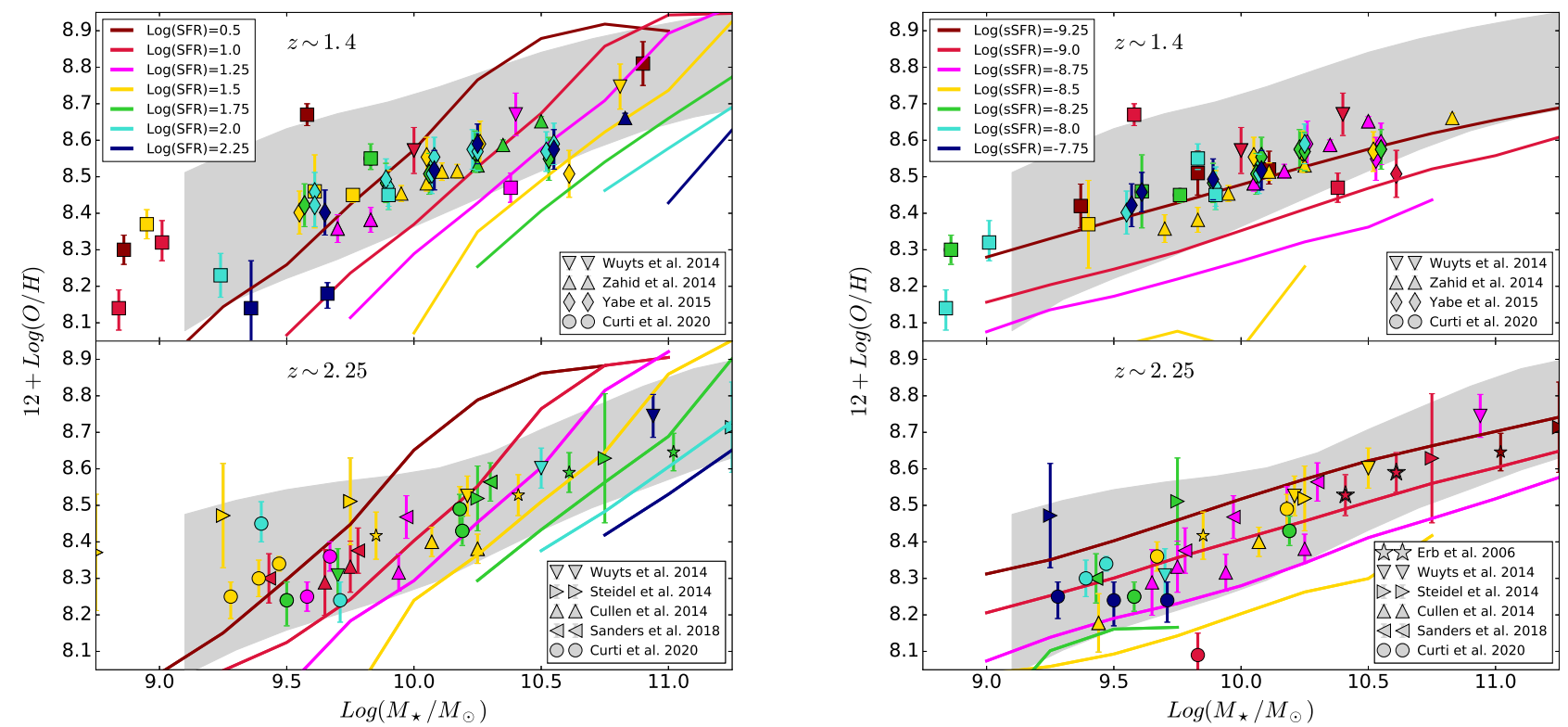

Figure 7. Gas Phase MZR at $1.4<z<2.25$. In the left (right) panel, both data and model predictions have been split in colour according to their SFR (sSFR) levels, as indicated in the legend. The grey shaded area shows the predicted MZR as in Fig. 1. Only data with SFR (sSFR) inside the considered model range are shown.

lations, the degree of predicted evolution is relatively small $(\sim 0.2$ dex), and of the same order of magnitude as the intrinsic scatter in observed samples at lower redshifts. Our model thus suggests that it will extremely difficult to constrain any evolutionary trend at these redshifts, even using JWST observations. Finally, in Fig. 4, we show the evolution of the gas-phase FMR in the redshift range $z \sim 4-5$. All $\Delta_{g}$ are still within 0.2 dex from the $z=0$ FMR, thus showing that this scaling relation is already well defined in GAEA up to the highest redshift.

\section{DISCUSSION}

In previous sections, we discussed the MZRs and the FMR (and their redshift evolution). In Fig. 5 we consider the same data as in Fig. 1, but we show the MZR at fixed SFR (as marked in the legend). The MZR derives from the linear convolution of these individual relations, weighted by the space densities of galaxies with the corresponding SFR. The difference between the individual lines shown in Fig. 5 and the global relations show in Fig. 1 (especially at high-redshifts) reflects the redshift evolution of the relative contribution of galaxies at fixed SFR. Fig. 5 clearly shows that GAEA predicts a MZR at fixed SFR much steeper than the global MZR, for both the stellar and gaseous components. This is particularly evident for the gas-phase, where none of the individual MZR at fixed SFR has a slope similar to that of the global MZR. Observational measurements of the FMR (see e.g. Curti et al. 2020a, their Fig. 6) indeed favour steeper slopes for high-SFR galaxies; the slope then shallows, becoming compatible with the slope of the global MZR below $1 \mathrm{M}_{\odot} \mathrm{yr}^{-1}$. The invariance of the MZR slope in GAEA at fixed SFR seems to suggest that the balance between gas cooling, star formation and feedback processes, as modeled by the SAM, works at all stellar mass scales (see e.g. Finlator \& Davé 2008).
The observed slope evolution may thus hint that either a different equilibrium configuration is in place at different mass scales or that additional physical processes are at play for low-mass galaxies. Another possible caveat is related to the fact that in Fig. 5 and 7 we are showing the intrinsic GAEA predictions: convolving these with an estimate of the typical error in the SFR and $M_{\star}$ determinations has the effect of flattening out the intrinsic relations (De Lucia et al. 2020), thus easing the discrepancy with the data.

In Fig. 6, we consider the MZRs at fixed sSFR. When we split according to the sSFR, the MZRs are characterized by slopes more similar to the global MZR, both in the stellar and gas-phases. In particular, these plots clearly indicate the typical sSFR of the galaxy population dominating the MZRs. The slope of the individual MZR at fixed sSFR are pretty similar to each other, in reasonable agreement with the observational trends, that show only a small evolution (Curti et al. 2020a) with sSFR. The normalization of the MZR shows a decreasing trend at increasing sSFR, which brings the $\mathrm{SSFR}>10^{-8.5} \mathrm{yr}^{-1}$ relations in better agreement with the $\mathrm{z} \sim 3.5$ VANDELS measurements. It is thus tempting to explain the discrepancy between the VANDELS and GAEA MZR as an effect of the different typical sSFR of the underlying population. However, sSFR $>10^{-8.5} \mathrm{yr}^{-1}$ galaxies in GAEA represents a population well separated from the SF main sequence, as can be appreciated by comparing the MZR at fixed SSFR with the shaded area representing the range between 16th and 84th precentiles of the distribution. We do not expect VANDELS galaxies to be that systematically offset from the $\mathrm{z} \sim 3.5$ main sequence (Garilli et al. 2021).

In Fig. 5 and 6, we consider SFR and SSFR ranges wide enough to include all galaxies in our model sample. However, data at intermediate redshift $(1.4<z<2.5)$ allow for a direct comparison in a comparable SFR range. In the left panel of Fig. 7 we colour-coded the MZR at fixed SFR and we then mark with similar 
colour the position of individual galaxies (only data with SFR or sSFR inside the considered model range are shown). Whereas it is difficult to find a clear FMR trend from such a small galaxy sample, it is clear that GAEA predictions tend to underpredict galaxy metallicity at fixed SFR, especially at $M_{\star} \sim 10^{10} \mathrm{M}_{\odot}$. Furthermore, there is a clear difference in the stellar mass range covered at fixed SFR in model predictions and observations. While GAEA predicts a clear correlation between $M_{\star}$ and SFR (with the largest SFR available only for more massive galaxies), the range of observed $M_{\star}$ at fixed SFR is typically much larger and irrespective of the actual SFR value. This discrepancy may be connected to the different way of estimating SFR in GAEA and in the data. However, it is worth noting that the observational estimates are mainly based on $\mathrm{H}_{\alpha}$ lines that sample recent $\mathrm{SFR}\left(<10^{7} \mathrm{yrs}\right)$ that are comparable with the model SFRs, which are computed over the time-span between two snapshots in the simulations (roughly covering a few $10^{8}$ yrs). When considering the right panel of Fig. 7, the data do not show any clear trend in SSFR as GAEA predictions clearly do. In particular the model has a hard times reproducing the metallicity of the highest sSFR $\left(>10^{-8.5} \mathrm{yr}^{-1}\right)$ in moderate mass $<10^{10} \mathrm{M}_{\odot}$ galaxies. Of course, also in this case the different SFR estimate between data and models should play a role in the comparison.

As a final check, we also consider the stellar MZR for quiescent and star-forming galaxies (Peng et al. 2015; Trussler et al. 2020). In particular, in Fig. 8 we consider recent results using the SDSS sample from Gallazzi et al. (2021). They confirm a clear trend of lower stellar metallicity at $M_{\star}<10^{11} \mathrm{M}_{\odot}$ for star-forming galaxies with respect to quiescent sources. We then compare these data against GAEA predictions using the same sSFR cut as in Gallazzi et al. (2021, see figure legend for more details). At variance with SDSS data, GAEA predicts an almost indistinguishable MZR for quiescent and star-forming galaxies. We can understand these results in the light of our previous analysis: GAEA predicts no slope variation for the MZR at fixed sSFR, and the assumed activity threshold level at $z \sim 0$ is too low for the normalization between the resulting populations to be different. We thus interpret this result as the effect of the tension between the observed and predicted FMR, and in particular to the fact that the slope of the FMR at fixed SFR does not evolve in GAEA in the $M_{\star}<10^{11} \mathrm{M}_{\odot}$ mass range.

\subsection{Comparison with other theoretical models.}

In this section, we will put the results we discuss in this paper into the context of other recent theoretical models. The main problem in such a comparison lies in the fact that the different groups use different observational samples as benchmark to assess the reliability of their model MZR predictions. Given the uncertainties on the absolute normalization of MZR (relative to the different spectral features used), this translates into a limited quantitative comparison between the models.

Within the SAM framework, Yates et al. (2012) discuss the evolution of the MZRs in the context of the Guo et al. (2011) model: they find that the $z=0$ gas-phase MZR is well reproduced by model predictions, but it shows negligible evolution to higher redshifts. This is due to a rapid enrichment of the cold gas in the model, that brings galaxies onto the local relation already at $z \sim 3$. This behaviour is connected with the too efficient formation of $M_{\star}<10^{11} \mathrm{M}_{\odot}$ galaxies in previous generations of SAMs and hydro-simulations (see e.g. Fontanot et al. 2009; Weinmann et al. 2012). A more recent rendition of the model (Yates et al. 2021) shows a more relevant evolution of the MZR of the order to $0.3 \mathrm{dex}$ from $z \sim 3$ to $z \sim 0$, as an effect of the assumed highly efficient metal removal from galaxies via outflows.

The evolution of the MZRs and their relation with the FMR is the focus of recent work in the framework of hydro-simulations. The EAGLE simulation (Schaye et al. 2015) is able to reproduce the local gas-phase MZR and FMR. Lagos et al. (2016) and De Rossi et al. (2017) further expand the analysis to higherredshifts, by focusing in particular on the redshift evolution of the gas-phase MZR. They find that the evolution of the gas-phase MZR is well reproduced up to $z \sim 1.5$. At higher redshifts the situation is less clear, and they show that EAGLE predicts too large metallicities at $z>3$ with respect to the observational estimates from Maiolino et al. (2008, and based to a subsample of the Troncoso et al. 2014 data used in this paper). However, they also point out that the predicted evolution is in better agreement with the observed evolution up to $z \sim 3$ in the Metallicity Evolution and Galaxy Assembly (MEGA) dataset (Hunt et al. 2016). Interestingly, they also find the FMR to be already established at $z \sim 5$, in agreement with our results.

Torrey et al. (2019) study the evolution of the gas-phase MZR in the context of the ILLUSTRISTNG simulation (Pillepich et al. 2018), and find that the model reproduces its slope and normalization evolution up to $z \lesssim 2$. At higher redshifts, the same considerations and caveats we discussed for EAGLE apply. An important aspect in the ILLUSTRISTNG framework lies in the fact that the model assumes that stellar-driven galactic winds are metal depleted with respect to their parent ISM. This choice originates from the assumption that they are hydro-dynamically decoupled for some time, in order to account for the entrainment of wind material with the lower-metallicity circum-galactic medium. At variance with this approach, stellar driven winds in GAEA retain the same metallicity as the ISM of the parent galaxy.

SIMBA (Davé et al. 2019) is a cosmological hydrodynamical simulation that implements a model for stellar feedback derived from the fitting formulae proposed in Muratov et al. (2015). In Cullen et al. (2019), we show that this realization underpredicts the stellar metallicity levels found in VANDELS $z \sim 3.5$ galaxies, although it correctly reproduces the shape of the observed MZR relation. Unfortunately, in that paper SIMBA was not run down to $z=0$, and no other datasets was available in the redshift range covered by the simulation, so that we cannot explicitly check the predicted evolution of the stellar MZR.

Cullen et al. (2019) also present a comparison of their stellar MZR with the corresponding relation defined by the FIRE suite of high-resolution zoom simulations (Ma et al. 2016), showing that these model galaxies correctly reproduce the amount of evolution from $z \sim 3-4$ to $z \sim 0$. It is important to keep in mind that the (Ma et al. 2016) sample is based on simulations of isolated dark matter haloes, therefore it lacks the statistical information on cosmological volumes. Nonetheless, the overall normalization of the theoretical MZRs has to be shifted upwards by 0.3 dex to match the actual observations, highlighting once more the difficulties of such models to reproduce the correct level of chemical enrichment in individual galaxies. Cullen et al. (2019) conclude that shape and normalization of the MZR in hydro-simulations are determined by the strength of galactic outflows: models consistent with the FIRE feedback scaling relations typically underpredicts the normalization of the MZR, while earlier schemes with weaker galactic wind (like for example Dalla Vecchia \& Schaye 2012) tend to overpredict galaxy metallicities at higher redshifts. Our results based on GAEA confirm on a cosmological volume that models implementing scaling relations for stellar feedback based on the FIRE simu- 


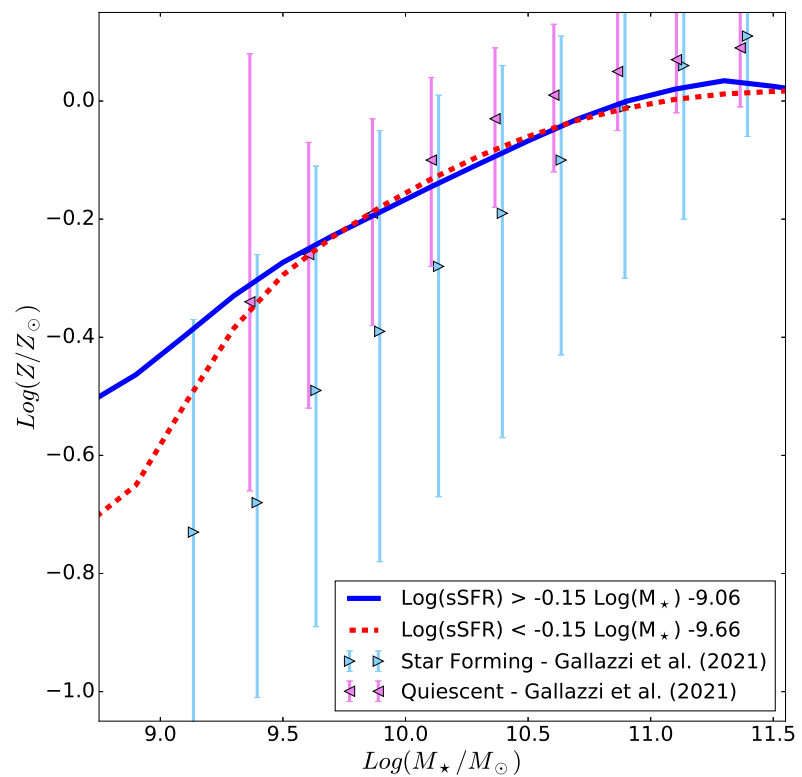

Figure 8. $z=0$ stellar MZR for star-forming and quiescent galaxies. Both SDSS data (triangles with errorbars) and model galaxies (colored lines) have been separated into star-forming and quiescent samples using the sSFR cuts defined in Gallazzi et al. (2021) and indicated in the legend. Errobars represent the 16th and 84th percentiles of the distribution.

lations are indeed able to provide a reliable prediction for the evolution of the MZR relations (both in stellar and gas-phase). Moreover, we show that our prescriptions calibrated on reproducing the GSMF are also able to recover the normalization of the MZR to within $\sim 0.1$ dex.

\section{SUMMARY}

In this paper, we present the comparison of the predictions of the semi-analytic model GAEA with the $z \sim 3.5$ stellar MZR measured in the VANDELS framework. In order to put these results in the broader context of galaxy formation and evolution we also consider other determinations of the stellar MZR at lower redshift and the redshift evolution of gas-phase MZR at $z<3.5$. Finally, we also consider the evolution of the FMR defined by the 3-dimensional $M_{\star}$, SFR and metallicity space.

We show that GAEA is able to reproduce the evolution of the gas-phase MZR from $z \sim 3.5$ to present, although some recalibration of model predictions might be required to match the absolute scale without resorting to a constant shift in the overall normalization. However, this small shift is perfectly in line with the uncertainty in the observational determination of metallicities. The agreement between model predictions and the observed stellar MZR is good up to $z \sim 0.7$, while some tensions (at 2- $\sigma$ level) with the results of the VANDELS sample are evident at $z \sim 3.5$, in terms of both the slope and normalization of the stellar MZR. A likely solution for the slope tension lies in the selection criteria adopted in VANDELS. If VANDELS target have been selected with a SFR higher than the typical value in GAEA, that may explain the different slope for the MZR (Calabrò et al. 2021). The different normalization of the MZR can be partly explained by the fact that $Z_{\star}$ estimates in VANDELS are FUV-weighted and this may lead to an offset to lower metallicities with respect to mass-weighted metallicities (Cullen et al. 2019). A more quantitative comparison between model predictions and observational determination requires to expand GAEA in order to predict realistic synthetic spectra including all the relevant spectral features for metallicity determination in the different phases (see e.g. Hirschmann et al. 2017, 2019). These synthetic features could be than processed in the same way as the real data, and provide fundamental clues on the relation between intrinsic and measured metallicities. We plan to explore these ideas in forthcoming works.

Furthermore, GAEA predicts a steeper slope for the MZR at fixed SFR, i.e. the FMR. As shown in De Lucia et al. (2020), these predictions are in reasonable agreement with the observational determinations (Curti et al. 2020a) for high-SFR galaxies, while some tension arise for sources with lower-levels of activity: below a few $\mathrm{M}_{\odot} \mathrm{yr}^{-1}$ data suggest a flattening of the slope of the MZR at fixed SFR, while GAEA predicts a constant slope (and steeper than the overall MZR). This is probably the strongest discrepancy between model predictions and the available observational constraints. However, it is also worth noting that a possible way to reconcile data and models lies in considering the effect of the uncertainties in the SFR determination, when estimating the slope of the relation. Larger SFR errors may lead to flatter slopes than the intrinsic value, so that an increasing error at increasing SFR would mimic a slope evolution.

Furthermore, we use GAEA realizations to explore the redshift evolution of the FMR. GAEA predicts the gas-phase FMR to be already well established at $z \sim 5$ : this implies that the observed evolution of the gas-phase MZR are mainly driven by typical galaxies at a given redshift populating different regions of the FMR. On the other hand, the stellar FMR shows a marked evolution in its normalization, with galaxies increasing their $Z_{\star}$ content with cosmic time. These predictions will be challenged with future facilities like JWST, which will be able to open the $z \gtrsim 4$ window to metallicity studies thanks to spectral range covered by the NIRSPEC instrument. In the meantime, stellar metallicity studies using some of the most recent Multi-Object Spectrograph facilities (such as WEAVE, 4MOST, MOONS, MOSAIC) or surveys like LEGA-C (van der Wel et al. 2016) will be able to close the gap at intermediate to high redshifts and give us a better understanding of the MZR evolution at $1 \lesssim z \lesssim 3$, also taking into account the known uncertainties in the derivation of stellar metallicities from spectral analysis.

These results confirm GAEA is able to reproduce, at the same time, the evolution of the GSMF up to $z \sim 7$, the cosmic SFR up to $z \sim 10$ and the evolution of the stellar content up to $z \sim 3.5$. This is an important confirmation for our approach to stellar feedback, highlighting once more the relevance of stellar-driven winds in shaping galaxy evolution.

\section{ACKNOWLEDGEMENTS}

This work is based on data products from observations made with ESO Telescopes at La Silla Paranal Observatory under ESO programme ID 194.A-2003 (PIs: Laura Pentericci and Ross McLure) and ESO Large Program 197.A-0717 (PI: Michele Cirasuolo). RA acknowledges support from ANID FONDECYT Regular Grant 1202007. 


\section{DATA AVAILABILITY}

An introduction to GAEA, a list of our recent work, as well as datafile containing published model predictions, can be found at http://adlibitum.oats.inaf.it/delucia/GAEA/. In particular, the predictions used in this paper will be shared on request to the corresponding author. The latest VANDELS data release is available through our public database at http://vandels.inaf.it/dr3.html or via the ESO archive.

\section{REFERENCES}

Blitz L., Rosolowsky E., 2006, ApJ, 650, 933

Bothwell M. S., Maiolino R., Kennicutt R., Cresci G., Mannucci F., Marconi A., Cicone C., 2013, MNRAS, 433, 1425

Bothwell M. S., Maiolino R., Peng Y., Cicone C., Griffith H., Wagg J., 2016, MNRAS, 455, 1156

Bresolin F., Kudritzki R.-P., Urbaneja M. A., Gieren W., Ho I. T., Pietrzyński G., 2016, ApJ, 830, 64

Brown T., Cortese L., Catinella B., Kilborn V., 2018, MNRAS, 473, 1868

Calabrò A., et al., 2021, A\&A, 646, A39

Chieffi A., Limongi M., 2002, ApJ, 577, 281

Cresci G., Mannucci F., Sommariva V., Maiolino R., Marconi A., Brusa M., 2012, MNRAS, 421, 262

Cresci G., Mannucci F., Curti M., 2019, A\&A, 627, A42

Cullen F., Cirasuolo M., McLure R. J., Dunlop J. S., Bowler R. A. A., 2014, MNRAS, 440, 2300

Cullen F., et al., 2019, MNRAS, 487, 2038

Curti M., Mannucci F., Cresci G., Maiolino R., 2020a, MNRAS, 491, 944

Curti M., et al., 2020b, MNRAS, 492, 821

Dalla Vecchia C., Schaye J., 2012, MNRAS, 426, 140

Davé R., Anglés-Alcázar D., Narayanan D., Li Q., Rafieferantsoa M. H., Appleby S., 2019, MNRAS, 486, 2827

Dayal P., Ferrara A., Dunlop J. S., 2013, MNRAS, 430, 2891

De Lucia G., Tornatore L., Frenk C. S., Helmi A., Navarro J. F., White S. D. M., 2014, MNRAS, 445, 970

De Lucia G., Hirschmann M., Fontanot F., 2019, MNRAS, 482, 5041

De Lucia G., Xie L., Fontanot F., Hirschmann M., 2020, MNRAS, 498, 3215

De Rossi M. E., Bower R. G., Font A. S., Schaye J., Theuns T., 2017, MNRAS, 472, 3354

Ellison S. L., Patton D. R., Simard L., McConnachie A. W., 2008, ApJ, 672, L107

Erb D. K., Shapley A. E., Pettini M., Steidel C. C., Reddy N. A., Adelberger K. L., 2006, ApJ, 644, 813

Ferreras I., et al., 2009, ApJ, 706, 158

Finlator K., Davé R., 2008, MNRAS, 385, 2181

Fontanot F., De Lucia G., Monaco P., Somerville R. S., Santini P., 2009, MNRAS, 397, 1776

Fontanot F., Hirschmann M., De Lucia G., 2017, ApJ, 842, L14

Gallazzi A., Charlot S., Brinchmann J., White S. D. M., Tremonti C. A., 2005, MNRAS, 362, 41

Gallazzi A., Bell E. F., Zibetti S., Brinchmann J., Kelson D. D., 2014, ApJ, 788, 72

Gallazzi A. R., Pasquali A., Zibetti S., Barbera F. L., 2021, MNRAS, 502,4457

Garilli B., et al., 2021, A\&A, 647, A150

Grogin N. A., Kocevski D. D., Faber S. M., Ferguson H. C., Koekemoer A. M., Riess A. G., Acquaviva V., Alexander D. M. e. a., 2011, ApJS, 197, 35

Guo Q., et al., 2011, MNRAS, 413, 101

Guo Q., White S., Angulo R. E., Henriques B., Lemson G., Boylan-Kolchin M., Thomas P., Short C., 2013, MNRAS, 428, 1351

Henriques B. M. B., White S. D. M., Thomas P. A., Angulo R. E., Guo Q., Lemson G., Springel V., 2013, MNRAS, 431, 3373
Hirschmann M., De Lucia G., Fontanot F., 2016, MNRAS, 461, 1760

Hirschmann M., Charlot S., Feltre A., Naab T., Choi E., Ostriker J. P., Somerville R. S., 2017, MNRAS, 472, 2468

Hirschmann M., Charlot S., Feltre A., Naab T., Somerville R. S., Choi E., 2019, MNRAS, 487, 333

Hunt L., et al., 2012, MNRAS, 427, 906

Hunt L., Dayal P., Magrini L., Ferrara A., 2016, MNRAS, 463, 2002

Karakas A. I., 2010, MNRAS, 403, 1413

Kewley L. J., Ellison S. L., 2008, ApJ, 681, 1183

Koekemoer A. M., Faber S. M., Ferguson H. C., Grogin N. A., Kocevski D. D., Koo D. C., Lai K., Lotz J. M. e. a., 2011, ApJS, 197, 36

Lagos C. d. P., et al., 2016, MNRAS, 459, 2632

Lara-López M. A., et al., 2010, A\&A, 521, L53

Ma X., Hopkins P. F., Faucher-Giguère C.-A., Zolman N., Muratov A. L., Kereš D., Quataert E., 2016, MNRAS, 456, 2140

Maiolino R., Mannucci F., 2019, A\&A Rev., 27, 3

Maiolino R., Nagao T., Grazian A., Cocchia F., Marconi A., Mannucci F., Cimatti A., Pipino A. e. a., 2008, A\&A, 488, 463

Mannucci F., et al., 2009, MNRAS, 398, 1915

Mannucci F., Cresci G., Maiolino R., Marconi A., Gnerucci A., 2010, MNRAS, 408, 2115

McLure R. J., et al., 2018, MNRAS, 479, 25

Muratov A. L., Kereš D., Faucher-Giguère C.-A., Hopkins P. F., Quataert E., Murray N., 2015, MNRAS, 454, 2691

Namiki S. V., et al., 2019, ApJ, 877, 118

Nelson D., et al., 2018, MNRAS, 475, 624

Onodera M., et al., 2016, ApJ, 822, 42

Padovani P., Matteucci F., 1993, ApJ, 416, 26

Pasquali A., Gallazzi A., van den Bosch F. C., 2012, MNRAS, 425, 273

Peng Y., Maiolino R., Cochrane R., 2015, Nature, 521, 192

Pentericci L., et al., 2018, A\&A, 616, A174

Pérez-Montero E., 2014, MNRAS, 441, 2663

Pérez-Montero E., et al., 2013, A\&A, 549, A25

Pillepich A., et al., 2018, MNRAS, 473, 4077

Pilyugin L. S., Vílchez J. M., Thuan T. X., 2010, ApJ, 720, 1738

Sanders R. L., et al., 2018, ApJ, 858, 99

Sanders R. L., et al., 2020, preprint, (arXiv:2009.07292)

Schaye J., et al., 2015, MNRAS, 446, 521

Somerville R. S., Davé R., 2015, ARA\&A, 53, 51

Sommariva V., Mannucci F., Cresci G., Maiolino R., Marconi A., Nagao T., Baroni A., Grazian A., 2012, A\&A, 539, A136

Spitoni E., Vincenzo F., Matteucci F., 2017, A\&A, 599, A6

Springel V., et al., 2005, Nature, 435, 629

Steidel C. C., et al., 2014, ApJ, 795, 165

Talia M., et al., 2021, in preparation

Thielemann F. K., et al., 2003, Nucl. Phys. A, 718, 139

Torrey P., et al., 2019, MNRAS, 484, 5587

Tremonti C. A., et al., 2004, ApJ, 613, 898

Troncoso P., et al., 2014, A\&A, 563, A58

Trussler J., Maiolino R., Maraston C., Peng Y., Thomas D., Goddard D., Lian J., 2020, MNRAS, 491, 5406

Tsamis Y. G., Barlow M. J., Liu X. W., Danziger I. J., Storey P. J., 2003, MNRAS, 345, 186

Weinmann S. M., Pasquali A., Oppenheimer B. D., Finlator K., Mendel J. T., Crain R. A., Macciò A. V., 2012, MNRAS, 426, 2797

Wuyts E., et al., 2014, ApJ, 789, L40

Xie L., De Lucia G., Hirschmann M., Fontanot F., Zoldan A., 2017, MNRAS, 469, 968

Xie L., De Lucia G., Hirschmann M., Fontanot F., 2020, MNRAS, 498,4327

Yabe K., et al., 2015, PASJ, 67, 102

Yates R. M., Kauffmann G., Guo Q., 2012, MNRAS, 422, 215

Yates R. M., Henriques B. M. B., Fu J., Kauffmann G., Thomas P. A., Guo Q., White S. D. M., Schady P., 2021, MNRAS, 503, 4474

Zahid H. J., Dima G. I., Kudritzki R.-P., Kewley L. J., Geller M. J., Hwang H. S., Silverman J. D., Kashino D., 2014a, ApJ, 791, 130

Zahid H. J., et al., 2014b, ApJ, 792, 75 
Zahid H. J., Kudritzki R.-P., Conroy C., Andrews B., Ho I. T., 2017, ApJ, 847,18

van der Wel A., et al., 2016, ApJS, 223, 29 